\title{
MOESP-type Closed-loop Subspace Model Identification Method
}

\author{
Hiroshi OKu*, Yasuko OGURA* and Takao FuJir**
}

\begin{abstract}
In this paper, a MOESP-type closed-loop subspace model identification method is developed. Although external exciting signals and a priori knowledge of a controller in closed-loop are required, the procedure of the proposed method is very similar to the procedures of the existing MOESP methods. Simulation examples are provided to compare the proposed method with some of the existing methods, that is, CCA ${ }^{10)}$, $\mathrm{SSARX}^{5)}$ and PO-MOESP ${ }^{4)}$, and demonstrate that the proposed method is capable of identification not only of an ARMAX model but of a Box-Jenkins model.
\end{abstract}

Key Words: subspace methods, closed-loop identification, identification algorithms, linear multivariable systems, feedback systems

\section{Introduction}

It is known that the existing MOESP methods ${ }^{1) ~ 4)}$ are not capable of dealing with closed-loop data. Actually, the numerical study by Jansson ${ }^{5}$ ) has shown that POMOESP ${ }^{4)}$ did not give a good performance on closed-loop identification.

However, in this paper, we will develop a MOESP-type closed-loop subspace model identification method. The procedure of the proposed method is very similar to those of the MOESP methods, among which the difference is in bunches of data Hankel matrices to be $Q R$-decomposed. Although it requires external exciting signals and a priori knowledge of a controller in closed-loop, the proposed method is unlike the closed-loop identification methods proposed by e.g., Verhaegen ${ }^{6)}$ and Katayama et al. ${ }^{7)}$, both of which use the joint input-output identification approach and require the model-reduction step.

There have been made several attempts on closed-loop identification using the direct identification approach. The SSARX method ${ }^{5)}$ is one of the most effective methods so far. However, SSARX requires the crucial assumption that a system to be identified must be modelled by an innovations form, which is related to ARMAX model structure. Numerical examples in Section 5 will illustrate that the performance of SSARX is significantly deteriorated when the assumption is not satisfied.

As shown in Section 4, the proposed method in this paper can be regarded as a modified version of the method

* Faculty of Engineering, Osaka Institute of Technology, 5-16-1, Omiya, Asahi-ku, Osaka

** Graduate School of Engineering Science, Osaka University, 1-3, Machikaneyamamachi, Toyonaka

(Received October 12, 2005)

(Revised January 13, 2006) previously proposed by the authors ${ }^{8)}$, which has been developed by integrating PI-MOESP ${ }^{3)}$ into the Two-stage method $^{9)}$. Therefore, why the method proposed here works effectively may be found in the Two-stage method. The numerical examples in Section 5 will demonstrate that the proposed method is applicable to not only ARMAX model structure but Box-Jenkins model structure.

The rest of the paper is organized as follows. After the problem formulation in Section 2, notations and assumptions are introduced, followed by a brief review of the closed-loop identification method previously proposed by the authors ${ }^{8)}$ in Section 3. Section 4 is the main part of the paper where the procedure of the MOESPtype closed-loop identification method are presented, followed by a theorem to verify the validity of the proposed method. In Section 5, simulation examples are provided to compare the proposed method with some of the existing methods, that is, $\mathrm{CCA}^{10)}, \mathrm{SSARX}^{5)}$ and PO-MOESP ${ }^{4)}$.

\section{Problem formulation}

The identification framework considered in this paper is the same as in the literature 8 ), which is very similar to those in the literature 9) and 11). Consider a closedloop system depicted in Fig. 1. Let us suppose that $C$ is a known stable linear system, and that the observed signals $u, r^{1} \in \mathbb{R}^{m}$ and $y, r^{2} \in \mathbb{R}^{l}$ are quasi-stationary ${ }^{11)}$. Define $r:=r^{1}+C r^{2}$. $G$ is the system to be estimated, which is supposed to be stable. Assume that the closed-loop system is internally stable. The disturbance signal $v \in \mathbb{R}^{l}$ is assumed to be a zero-mean white noise uncorrelated with $r$. $H$ is assumed to be stable and stably invertible. The task is to obtain a state space model which represents the input-output relation of the system $G$ in the closed-loop illustrated in Fig. 1 based on finite measurement data of 


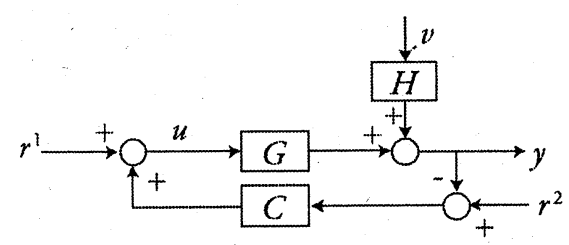

Fig. 1 Closed-loop system. $G$ is to be identified.

$r, u$ and $y$.

\section{Preliminaries}

\section{1 Notations and assumptions}

Let $W \in \mathbb{R}^{p \times q}$ and $X, Y \in \mathbb{R}^{r \times q}$. Similarly to the book 12 ), the orthogonal projections of the row space of $W$ on the row space of $X$ and onto the orthogonal complement of the row space of $X$ are, respectively, denoted by

$$
\begin{aligned}
W / X & :=W \Pi_{X}:=W X^{T}\left(X X^{T}\right)^{-1} X, \\
W / X^{\perp} & :=W \Pi_{X}^{\perp}:=W\left(I-X^{T}\left(X X^{T}\right)^{-1} X\right) .
\end{aligned}
$$

Note that, if $\left[X^{T} Y^{T}\right]^{T}$ is of full row rank,

$$
W /\left[\begin{array}{c}
X \\
Y
\end{array}\right]=W\left(\Pi_{X}+\Pi_{X}^{\perp} Y^{T}\left(Y \Pi_{X}^{\perp} Y^{T}\right)^{-1} Y \Pi_{X}^{\perp}\right)
$$

To indicate the partitioning of a matrix, we will adopt the notation used in the MATLAB package. For example, $U(1: l,:)$ denotes the first $l$ rows of a matrix $U$.

Given a sampled data sequence $\left\{u_{i}\right\}$, the block Hankel matrix of $s$ block rows, $\mathcal{U}_{i, j}$, is defined as

$$
\mathcal{U}_{i, j}:=\overbrace{\left[\begin{array}{cccc}
u_{i} & u_{i+1} & \cdots & u_{i+j-1} \\
u_{i+1} & u_{i+2} & \cdots & u_{i+j} \\
\vdots & \vdots & & \vdots \\
u_{i+s-1} & u_{i+s} & \cdots & u_{i+j+s-2}
\end{array}\right]}^{j \text { columns }}
$$

where the subscripts on $\mathcal{U}$, i.e., $i$ and $j$, respectively, denote the subscript of the first element of the first column and the number of columns. Given sampled data sequences $\left\{y_{i}\right\}$ and $\left\{r_{i}\right\}$, the block Hankel matrices, $\mathcal{Y}_{i, j}$ and $\mathcal{R}_{i, j}$, respectively, are defined in a manner similar to (4). Note that $s$ is a user-defined index and should be chosen to be greater than the orders of $G$ and $S:=(I+C G)^{-1}$.

Hereinafter, with $N \gg s$, if we have sampled sequences $\left\{r_{t}\right\}_{t=1}^{N+s-1},\left\{u_{t}\right\}_{t=1}^{N+s-1},\left\{y_{t}\right\}_{t=1}^{N+s-1}$ at hand, we will assume

$$
\left[\begin{array}{c}
\mathcal{U}_{1, N} \\
\mathcal{U}_{s+1, N}
\end{array}\right] /\left[\begin{array}{c}
\mathcal{R}_{s+1, N} \\
\mathcal{R}_{1, N}
\end{array}\right]
$$

is of full row rank.

\section{2 Review of a direct closed-loop subspace model identification method}

In this subsection, we will briefly review a direct closed-loop subspace model identification method, named 'TS4SID' for convenience' sake, proposed by the authors $^{8)}$.

According to van den Hof and Schrama ${ }^{9)}$, the relations from $(r, v)$ to $(u, y)$ in Fig. 1 can be given by

$$
\begin{aligned}
& u=(I+C G)^{-1} r-(I+C G)^{-1} C H v \\
& y=G(I+C G)^{-1} r+(I+G C)^{-1} H v .
\end{aligned}
$$

They have pointed out that the closed-loop identification problem considered here can be reduced to two successive open-loop identification problems, i.e., one open-loop identification, from $r$ to $u$, of an output error model with a colored noise followed by the other open-loop identification, from $(I+C G)^{-1} r$ to $y$, of an output error model with a colored noise. It is called the Two-stage method.

In the literature 8), the authors have shown that the integration of the PI-MOESP method ${ }^{3)}$ into the Two-stage method $^{9)}$ has led to the TS4SID method. The algorithm presented below is slightly modified from the original one in the literature 8) from the view point of orthogonal projection.

Algorithm 1 (TS4SID ${ }^{8)}$ ). When we have a set of data $\left\{r_{t}\right\}_{t=1}^{N+s-1},\left\{u_{t}\right\}_{t=1}^{N+s-1},\left\{y_{t}\right\}_{t=1}^{N+s-1}$ at hand sampled from the closed-loop system depicted in Fig. 1, a state space model which represents the input-output relation of $G$ can be estimated according to the procedure as follows:

1. Compute the orthogonal projection of $\mathcal{U}_{s+1, N}$ onto the joint matrix $\left[\begin{array}{c}\mathcal{R}_{s+1, N} \\ \mathcal{R}_{1, N}\end{array}\right]$, denoted by $\mathcal{Z}_{s+1, N}$, and we have

$$
\mathcal{Z}_{s+1, N}:=\mathcal{U}_{s+1, N} /\left[\begin{array}{c}
\mathcal{R}_{s+1, N} \\
\mathcal{R}_{1, N}
\end{array}\right]=\mathcal{U}_{s+1, N} \Pi .
$$

Note that the projection matrix can be written by

$$
\begin{aligned}
\Pi:= & \Pi_{\mathcal{R}_{s+1, N}}+\Pi_{\mathcal{R}_{s+1, N}}^{\perp} \mathcal{R}_{1, N}^{T} \\
& \cdot\left(\mathcal{R}_{1, N} \Pi_{\mathcal{R}_{s+1, N}}^{\perp} \mathcal{R}_{1, N}^{T}\right)^{-1} \mathcal{R}_{1, N} \Pi_{\mathcal{R}_{s+1, N}}^{\perp} .
\end{aligned}
$$

2. It should be noted that $\mathcal{Z}_{s+1, N}$ is not a Hankel matrix like (4) any longer. When $\mathcal{Z}_{s+1, N}$ is denoted by

$$
\mathcal{Z}_{s+1, N}:=\left[\begin{array}{ccc}
z_{1,1} & \cdots & z_{1, N} \\
\vdots & & \vdots \\
z_{s, 1} & \cdots & z_{s, N}
\end{array}\right],
$$

$\mathcal{Z}_{1, N-s}$ and $\mathcal{Z}_{s+1, N-s}$ are, respectively, defined by 


$$
\begin{aligned}
\mathcal{Z}_{1, N-s} & :=\left[\begin{array}{ccc}
z_{1,1} & \cdots & z_{1, N-s} \\
\vdots & & \vdots \\
z_{s, 1} & \cdots & z_{s, N-s}
\end{array}\right], \\
\mathcal{Z}_{s+1, N-s} & :=\left[\begin{array}{ccc}
z_{1, s+1} & \cdots & z_{1, N} \\
\vdots & & \vdots \\
z_{s, s+1} & \cdots & z_{s, N}
\end{array}\right] .
\end{aligned}
$$

3. According to PI-MOESP method ${ }^{3)}$, QR decomposition of

$$
\left[\begin{array}{c}
\mathcal{Z}_{s+1, N-s} \\
\mathcal{Z}_{1, N-s} \\
\mathcal{Y}_{s+1, N-s}
\end{array}\right]=\left[\begin{array}{lll}
L_{11} & & \\
L_{21} & L_{22} & \\
L_{31} & L_{32} & L_{33}
\end{array}\right]\left[\begin{array}{c}
Q_{1}^{T} \\
Q_{2}^{T} \\
Q_{3}^{T}
\end{array}\right]
$$

followed by SVD of $L_{32}$, yields an estimate of the extended observability matrix $\mathcal{O}$ of the open-loop system $G$, and further an estimate of the quadruple $(A, B, C, D)$.

Note. It is known that

$$
\begin{aligned}
L_{32} Q_{2}^{T}= & \mathcal{Y}_{s+1, N-s} \Pi_{\mathcal{Z}_{s+1, N-s}}^{\perp} \mathcal{Z}_{1, N-s}^{T} \\
& \cdot\left(\mathcal{Z}_{1, N-s} \Pi_{\mathcal{Z}_{s+1, N-s}}^{\perp} \mathcal{Z}_{1, N-s}^{T}\right)^{-1} \\
& \cdot \mathcal{Z}_{1, N-s} \Pi_{\mathcal{Z}_{s+1, N-s}}^{\perp} \\
= & \mathcal{Y}_{s+1, N-s} /\left(\mathcal{Z}_{1, N-s} \Pi_{\mathcal{Z}_{s+1, N-s}}^{\perp}\right) .
\end{aligned}
$$

Note. It is worthwhile to point out that the orthogonal projection of $\mathcal{U}_{s+1, N}$ to $\left[\mathcal{R}_{s+1, N}^{T} \mathcal{R}_{1, N}^{T}\right]^{T}$ in the step 1 of the above algorithm exerts an effect approximately equivalent to filtering the input $u_{t}$ through an estimate of the sensitivity $S$ in the Two-stage $\operatorname{method}^{9)}$.

\section{MOESP-type closed-loop identification method}

Inspired by the step 1 of the TS4SID algorithm in the previous section, the idea is that, instead of the matrices $\mathcal{Z}_{1, N-s}$ and $\mathcal{Z}_{s+1, N-s}$, we adopt

$$
\begin{aligned}
& Z_{1, N}:=\mathcal{U}_{1, N} /\left[\begin{array}{c}
\mathcal{R}_{s+1, N} \\
\mathcal{R}_{1, N}
\end{array}\right], \\
& Z_{s+1, N}:=\mathcal{U}_{s+1, N} /\left[\begin{array}{c}
\mathcal{R}_{s+1, N} \\
\mathcal{R}_{1, N}
\end{array}\right]\left(=\mathcal{Z}_{s+1, N}\right),
\end{aligned}
$$

and then, we compute the orthogonal projection of $\mathcal{Y}_{s+1, N}$ to the row space of $Z_{1, N} \Pi_{Z_{s+1, N}}^{\perp}$. The algorithm presented below is the main result of this paper.

Algorithm 2. When we have a set of data $\left\{r_{t}\right\}_{t=1}^{N+s-1},\left\{u_{t}\right\}_{t=1}^{N+s-1},\left\{y_{t}\right\}_{t=1}^{N+s-1}$ at hand sampled from the closed-loop system depicted in Fig. 1, a state space model which represents the input-output relation of $G$ can be obtained according to the procedure as follows:

1. Execute the QR-factorization of the following matrix:

$$
\left[\begin{array}{c}
\mathcal{R}_{1, N} \\
\mathcal{R}_{s+1, N} \\
\mathcal{U}_{1, N} \\
\mathcal{U}_{s+1, N} \\
\mathcal{Y}_{s+1, N}
\end{array}\right]=\left[\begin{array}{lllll}
\dot{L}_{11} & & & & \\
L_{21} & L_{22} & & & \\
L_{31} & L_{32} & L_{33} & & \\
L_{41} & L_{42} & L_{43} & L_{44} & \\
L_{51} & L_{52} & L_{53} & L_{54} & L_{55}
\end{array}\right]\left[\begin{array}{c}
Q_{1}^{T} \\
Q_{2}^{T} \\
Q_{3}^{T} \\
Q_{4}^{T} \\
Q_{5}^{T}
\end{array}\right]
$$

2. Compute

$$
\Upsilon^{\frac{1}{2}}:=\left(L_{51} P_{1}^{T}+L_{52} P_{2}^{T}\right)\left(P_{1} P_{1}^{T}+P_{2} P_{2}^{T}\right)^{-\frac{1}{2}},
$$

with $P_{j}(j=1,2)$ defined by

$$
\begin{aligned}
& P_{j}:=L_{3 j}-\left(L_{31} L_{41}^{T}+L_{32} L_{42}^{T}\right) \\
& \cdot\left(L_{41} L_{41}^{T}+L_{42} L_{42}^{T}\right)^{-1} L_{4 j} .
\end{aligned}
$$

3. To estimate the extended observability matrix of $G$, execute SVD of $\Upsilon^{\frac{1}{2}}$, and we have

$$
\left[\begin{array}{ll}
U & U^{\perp}
\end{array}\right]\left[\begin{array}{cc}
\Sigma & \\
& \tilde{\Sigma}
\end{array}\right]\left[\begin{array}{c}
V \\
V^{\perp}
\end{array}\right]^{T}=\Upsilon^{\frac{1}{2}},
$$

where the diagonal matrix $\Sigma \in \mathbb{R}^{n \times n}$ has $n$ dominant singular values as its diagonal entries, and $n$ corresponds to the order of $G$.

4. An estimate of the quadruple of $(A, B, C, D)$ of a state space representation of $G$ can be obtained according to the procedure similarly to the literature 1 ) as follows. $(A, C)$ are estimated by

$$
\begin{aligned}
& \hat{C}=U(1: l,:), \\
& \hat{A}=U(1: l(s-1),:)^{\dagger} U(l+1: l s,:),
\end{aligned}
$$

where $X^{\dagger}$ denotes the Moore-Penrose generalized inverse matrix of a matrix $X$. To estimate $(B, D)$, we define

$$
\begin{aligned}
\alpha & :=\left[\begin{array}{llll}
\alpha_{1} & \alpha_{2} & \cdots & \alpha_{s}
\end{array}\right]:=\left(U^{\perp}\right)^{T} \\
\beta & :=\left[\begin{array}{llll}
\beta_{1} & \beta_{2} & \cdots & \beta_{s}
\end{array}\right] \\
: & =\left(U^{\perp}\right)^{T}\left(L_{51} L_{41}^{T}+L_{52} L_{42}^{T}\right) \\
& \cdot\left(L_{41} L_{41}^{T}+L_{42} L_{42}^{T}\right)^{-1}
\end{aligned}
$$

and then, with $U_{1}:=U(1: l(s-1),:)$,

$$
\left[\begin{array}{c}
\hat{B} \\
\hat{D}
\end{array}\right]=\left[\begin{array}{cc}
I_{l} & 0 \\
0 & U_{1}
\end{array}\right]^{\dagger}\left[\begin{array}{cccc}
\alpha_{1} & \alpha_{2} & \cdots & \alpha_{s} \\
\alpha_{2} & & \alpha_{s} & \\
\vdots & \alpha_{s} & & \\
\alpha_{s} & & & O
\end{array}\right]^{\dagger}\left[\begin{array}{c}
\beta_{1} \\
\beta_{2} \\
\vdots \\
\beta_{s}
\end{array}\right] .
$$

The following theorem verifies the validity of the step 2 of the aforementioned algorithm:

Theorem 1. Define

$$
\Xi:=\mathcal{Y}_{s+1, N} /\left(Z_{1, N} \Pi_{Z_{s+1, N}}^{\perp}\right) .
$$


Then, we have

$$
\begin{aligned}
\Upsilon:= & \Xi \Xi^{T} \\
= & \left(L_{51} P_{1}^{T}+L_{52} P_{2}^{T}\right)\left(P_{1} P_{1}^{T}+P_{2} P_{2}^{T}\right)^{-1} \\
& \cdot\left(L_{51} P_{1}^{T}+L_{52} P_{2}^{T}\right)^{T} .
\end{aligned}
$$

Proof. See Appendix A.

Note. Note that

$$
\begin{aligned}
& P_{1} P_{1}^{T}+P_{2} P_{2}^{T} \\
&=\left(L_{31} L_{31}^{T}+L_{32} L_{32}\right)-\left(L_{31} L_{41}^{T}+L_{32} L_{42}^{T}\right) \\
& \cdot\left(L_{41} L_{41}^{T}+L_{42} L_{42}^{T}\right)^{-1}\left(L_{41} L_{31}^{T}+L_{42} L_{32}^{T}\right) .
\end{aligned}
$$

The invertivility of $P_{1} P_{1}^{T}+P_{2} P_{2}^{T}$ requires the assumption that $\left[\begin{array}{ll}L_{31} & L_{32} \\ L_{41} & L_{42}\end{array}\right]$ is of full row rank, since nonsingularity of the right hand side in (14) is equivalent to non-singularity of

$$
\begin{aligned}
& {\left[\begin{array}{cc}
L_{31} L_{31}^{T}+L_{32} L_{32}^{T} & L_{31} L_{41}^{T}+L_{32} L_{42}^{T} \\
L_{41} L_{31}^{T}+L_{42} L_{32}^{T} & L_{41} L_{41}^{T}+L_{42} L_{42}^{T}
\end{array}\right]} \\
& =\left[\begin{array}{ll}
L_{31} & L_{32} \\
L_{41} & L_{42}
\end{array}\right]\left[\begin{array}{cc}
L_{31}^{T} & L_{41}^{T} \\
L_{32}^{T} & L_{42}^{T}
\end{array}\right]
\end{aligned}
$$

The assumption is satisfied if (5) is of full row rank.

\section{Simulation examples}

\section{1 Example 1}

Consider a linear system operating in closed-loop depicted in Fig. 1, with

$$
\begin{aligned}
& G(z)=\frac{1}{1-1.6 z^{-1}+0.89 z^{-2}} \\
& C(z)=z^{-1}-0.8 z^{-2} \\
& H(z)=\frac{1-1.56 z^{-1}+1.045 z^{-2}-0.3338 z^{-3}}{\left(1-0.75 z^{-1}\right)\left(1-1.6 z^{-1}+0.89 z^{-2}\right)} .
\end{aligned}
$$

The setting of these transfer functions is borrowed from the literature ${ }^{9)}$. Note in this example that the poles of $H(z)$ include those of $G(z)$. The task is to identify the input-output relation of $G$ of (15). The external signals, $r^{1}, r^{2}, v$, are independent zero-mean unit-variance Gaussian random signals. For the Hankel matrices, $s=10$ is chosen. MATLAB ver. 7.0.4 on a Windows PC with Xeon $3.2 \mathrm{GHz}, 2 \mathrm{~GB}$ RAM is used.

In this simulation, 200 independent Monte Carlo experiments are used. In each simulation run, the second order state-space models are estimated based on $N+s-1=10000$ data samples of $r, u$ and $y$. For comparison, some of the existing methods, that is, TS4SID ${ }^{8)}$, $\mathrm{CCA}^{10)}$, SSARX $^{5)}$ and PO-MOESP ${ }^{4)}$ are studied. We must mention that CCA, SSARX and PO-MOESP requires $u$ and $y$ only, and that it is not necessary for $C$
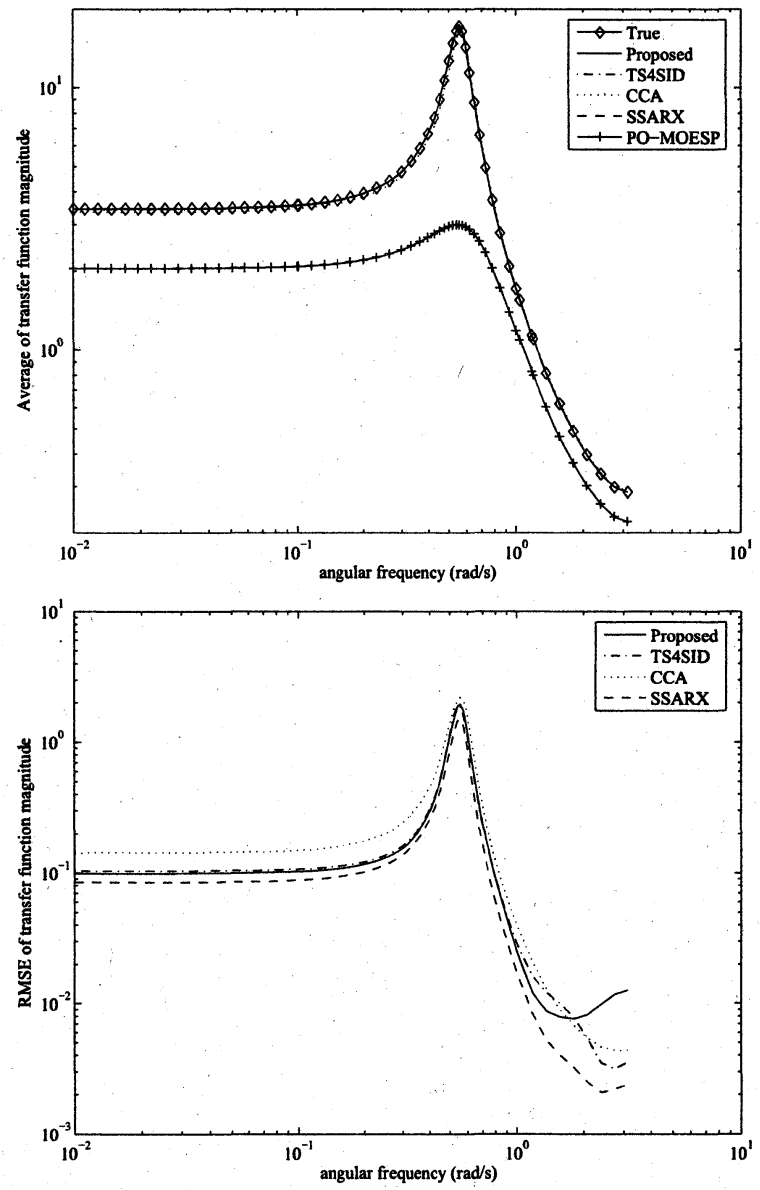

Fig. 2 Result of Example 1. The top graph shows the average of the 200 estimated magnitudes of the transfer function of $G$. The bottom graph shows the corresponding RMS errors of the estimated magnitudes.

to be known a priori. The user-defined indices for all the subspace methods are chosen to be 10 . The SMIToolbox ${ }^{13)}$ is used to implement PO-MOESP.

The top graph in Fig. 2 shows the average of the 200 estimated magnitudes of the transfer function of $G$. From the graph, it is obvious that PO-MOESP is the worst. The bottom graph shows the corresponding RMS errors of the estimated magnitudes, except for the RMS error of PO-MOESP. The performance of the proposed method looks better than that of CCA. TS4SID and the proposed method are comparable. However, we must admit that SSARX gives a better performance than the proposed method. Table 1 shows the averages and standard deviations of the CPU-times of 200 trials of the five methods. It is shown that the computation of the proposed method

Table 1 Average and standard deviation of CPU-time [s]

\begin{tabular}{llllll}
\hline & Proposed & TS4SID & CCA & SSARX & PO-MOESP \\
\hline Ave. & 0.1634 & 0.1103 & 0.1320 & 0.1684 & 0.1155 \\
Std. & 0.0099 & 0.0071 & 0.0096 & 0.0083 & 0.0083 \\
\hline
\end{tabular}


is not expensive.

\subsection{Example 2}

The difference between Example 1 and this example is only in the definition of $H(z)$, that is,

$$
H(z)=\frac{1-1.56 z^{-1}+1.045 z^{-2}-0.3338 z^{-3}}{\left(1-0.75 z^{-1}\right)\left(1+1.6 z^{-1}+0.89 z^{-2}\right)} .
$$

Taking it into account that 0.75 is significantly closer to the origin of the complex plane than the other poles of $H(z)$, it is important to point out that the input-output relation from $(u, v)$ to $y$ in Example 1 can approximately be described by an ARMAX model, while the inputoutput relation in this example is modelled by a BoxJenkins model.

From Fig. 3, it is found that the performance of the proposed method seems comparable to that in Example 1. This fact shows that the proposed method is applicable to closed-loop identification of a system modelled by a BoxJenkins model. This is a strong point of the proposed method. However, the performance of SSARX is significantly worse than that in Example 1. This fact supports the assumption on SSARX that the input-output relation of a system must be modelled by an innovations form ${ }^{5}$.

On the CPU-times of the five methods; the result is very similar to the one shown in Table 1 , and hence it is omitted.

\section{Concluding remarks}

In this paper, a MOESP-type closed-loop identification has been developed. The instruction on the proposed identification method has shown that this method is like the so-called MOESP family. The numerical study has told us an important fact as follows. When a system can (approximately) be modelled by an ARMAX model, SSARX proposed by Jansson ${ }^{5)}$ seems more reliable than the proposed method. However, when a system can be modelled by a Box-Jenkins model, the proposed method works satisfactorily while SSARX works poorly. To be precise, with respect to the proposed method, the RootMean-Square error in Example 2 is comparable to that in Example 1. This means that the proposed method is applicable to closed-loop identification without regard to the model structure, ARMAX or Box-Jenkins, with the help of the auxiliary information, i.e., the a priori knowledge of $C$ and the signal $r$. With respect to SSARX, it is the crucial assumption that a system to be identified must be modelled by an innovations form. Note that this assumption imposes us on $G$ and $H$ in Fig. 1 having the common poles. Note also that, generally speaking, the noise model
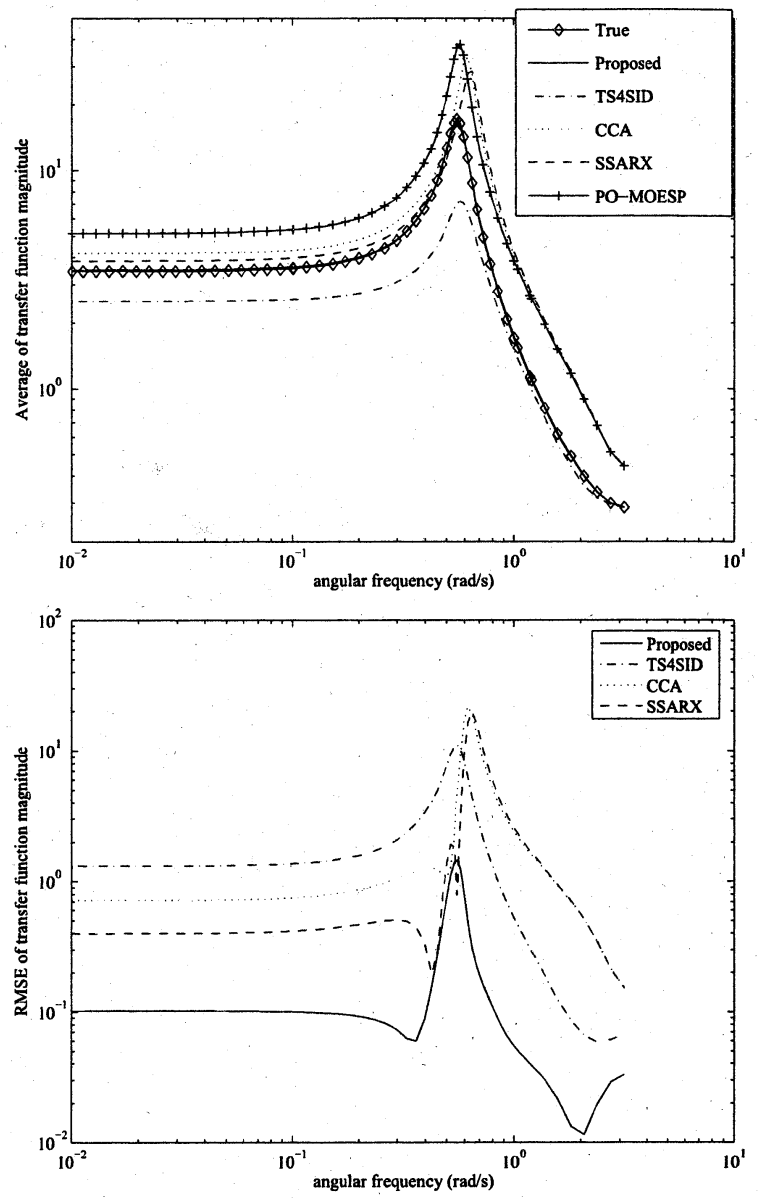

Fig. 3 Result of Example 2. The top graph shows the average of the 200 estimated magnitudes of the transfer function of $G$. The bottom graph shows the corresponding RMS errors of the estimated magnitudes.

$H$ should be unknown and that it is not necessary for $H$ and $G$ to have the common poles.

On derivation of a recursive algorithm of the proposed method, $L$-matrix in (10) can recursively be updated using a way similar to e.g., the recursive algorithm proposed by Verhaegen and Deprettere ${ }^{14)}$. This fact implies that the resultant recursive algorithm will be applicable to identification of slowly time-varying systems when an exponential forgetting factor is introduced.

\section{Acknowledgment}

This work was supported in part by The Ministry of Education, Culture, Sports, Science and Technology, Grantin-Aid for Young Scientists (B), Project No. 16760353.

\section{Appendix A. Proof of Theorem 1}

Hereinafter, we will adopt the following notations for notational brevity:

$$
\mathcal{R}_{p}:=\mathcal{R}_{1, N}, \quad \mathcal{R}_{f}:=\mathcal{R}_{s+1, N}, \quad \mathcal{U}_{p}:=\mathcal{U}_{1, N},
$$




$$
\begin{aligned}
& \mathcal{U}_{f}:=\mathcal{U}_{s+1, N}, \quad Z_{p}:=Z_{1, N}, \quad Z_{f}:=Z_{s+1, N} \\
& \mathcal{Y}_{f}:=\mathcal{Y}_{s+1, N} .
\end{aligned}
$$

Note that

$$
\Xi=\mathcal{Y}_{f} /\left(Z_{p} \Pi_{Z_{f}^{\perp}}\right)=\mathcal{Y}_{f} /\left(Z_{p}-Z_{p} \Pi_{Z_{f}}\right)
$$

For the proof, first we will express $Z_{f}, Z_{p}$ and $Z_{p} \Pi_{Z_{f}}$ by $L_{i j}$ and $Q_{i}$ in (10), then we will obtain $\Xi$ and $\Upsilon$. Throughout the proof, we will keep it in our mind that, for $i, j=1, \cdots, 5$,

$$
Q_{i}^{T} Q_{j}= \begin{cases}I, & i=j \\ 0, & i \neq j .\end{cases}
$$

From (10),

$$
\begin{aligned}
& \mathcal{R}_{p}=L_{11} Q_{1}^{T}, \quad \mathcal{R}_{f}=L_{21} Q_{1}^{T}+L_{22} Q_{2}^{T}, \\
& \mathcal{U}_{f}=L_{41} Q_{1}^{T}+L_{42} Q_{2}^{T}+L_{43} Q_{3}^{T}+L_{44} Q_{4}^{T} .
\end{aligned}
$$

From (A. 2),

$$
\begin{aligned}
&\left(\mathcal{R}_{f} \mathcal{R}_{f}^{T}\right)^{-1}=L_{22}^{-T} L_{22}^{-1}-L_{22}^{-T} L_{22}^{-1} L_{21} \cdot\left(I+L_{21}^{T} L_{22}^{-T} L_{22}^{-1} L_{21}\right)^{-1} L_{21}^{T} L_{22}^{-T} L_{22}^{-1} \\
& \Pi_{\mathcal{R}_{f}}=\mathcal{R}_{f}^{T}\left(\mathcal{R}_{f} \mathcal{R}_{f}^{T}\right)^{-1} \mathcal{R}_{f} \\
&=Q_{1} Q_{1}^{T}+Q_{2} Q_{2}^{T}-\left(Q_{1}-Q_{2} L_{22}^{-1} L_{21}\right) \\
& \cdot\left(I+L_{21}^{T} L_{22}^{-T} L_{22}^{-1} L_{21}\right)^{-1} \\
& \cdot\left(Q_{1}^{T}-L_{21}^{T} L_{22}^{-T} Q_{2}^{T}\right),
\end{aligned}
$$

and then,

$$
\begin{aligned}
\Pi_{\mathcal{R}_{f}}^{\perp} \mathcal{R}_{p}^{T}=\left(Q_{1}-Q_{2} L_{22}^{-1} L_{21}\right) \\
\cdot\left(I+L_{21}^{T} L_{22}^{-T} L_{22}^{-1} L_{21}\right)^{-1} L_{1}^{T}(\mathrm{~A} .5)
\end{aligned}
$$

From (A. 5), we have

$$
\left(\mathcal{R}_{p} \Pi_{\mathcal{R}_{f}^{\perp}} \mathcal{R}_{p}^{T}\right)^{-1}=L_{11}^{-T}\left(I+L_{21}^{T} L_{22}^{-T} L_{22}^{-1} L_{21}\right) L_{11}^{-1},(
$$

and then, from (A. 5) and (A.6),

$$
\begin{aligned}
& \Pi_{\mathcal{R}_{f}^{\perp}} \mathcal{R}_{p}^{T}\left(\mathcal{R}_{p} \Pi_{\mathcal{R}_{f}^{\perp}} \mathcal{R}_{p}^{T}\right)^{-1} \mathcal{R}_{p} \Pi_{\mathcal{R}_{f}^{\perp}} \\
& =\left(Q_{1}-Q_{2} L_{22}^{-1} L_{21}\right)\left(I+L_{21}^{T} L_{22}^{-T} L_{22}^{-1} L_{21}\right)^{-1} \\
& \quad\left(Q_{1}^{T}-L_{21}^{T} L_{22}^{-T} Q_{2}^{T}\right) .
\end{aligned}
$$

Therefore, from (A.4) and (A. 7), we have

$$
\begin{aligned}
& \Pi_{\mathcal{R}_{f}}+\Pi_{\mathcal{R}_{f}^{\perp}} \mathcal{R}_{p}^{T}\left(\mathcal{R}_{p} \Pi_{\mathcal{R}_{f}^{\perp}} \mathcal{R}_{p}^{T}\right)^{-1} \mathcal{R}_{p} \Pi_{\mathcal{R}_{f}^{\perp}} \\
& =Q_{1} Q_{1}^{T}+Q_{2} Q_{2}^{T}
\end{aligned}
$$

According to (3), the orthogonal projection of $\mathcal{U}_{f}$ to the joint matrix $\left[\begin{array}{ll}\mathcal{R}_{f}^{T} & \mathcal{R}_{p}^{T}\end{array}\right]^{T}$ can be obtained from (A.3) and (A. 8) as follows:

$$
Z_{f}=\mathcal{U}_{f} /\left[\begin{array}{c}
\mathcal{R}_{f} \\
\mathcal{R}_{p}
\end{array}\right]=L_{41} Q_{1}^{T}+L_{42} Q_{2}^{T}
$$

Similarly to (A.9), using (A. 8) and

$$
\mathcal{U}_{p}=L_{31} Q_{1}^{T}+L_{32} Q_{2}^{T}+L_{33} Q_{3}^{T}
$$

in (10), we have the orthogonal projection:

$$
Z_{p}=\mathcal{U}_{p} /\left[\begin{array}{c}
\mathcal{R}_{f} \\
\mathcal{R}_{p}
\end{array}\right]=L_{31} Q_{1}^{T}+L_{32} Q_{2}^{T}
$$

From (A. 9), we have

$$
\left(Z_{f} Z_{f}^{T}\right)^{-1}=\left(L_{41} L_{41}^{T}+L_{42} L_{42}^{T}\right)^{-1}
$$

and then,

$$
\begin{aligned}
\Pi_{Z_{f}}= & Z_{f}^{T}\left(Z_{f} Z_{f}^{T}\right)^{-1} Z_{f} \\
= & \left(Q_{1} L_{41}^{T}+Q_{2} L_{42}^{T}\right)\left(L_{41} L_{41}^{T}+L_{42} L_{42}^{T}\right)^{-1} \\
& \cdot\left(L_{41} Q_{1}^{T}+L_{42} Q_{2}^{T}\right) .
\end{aligned}
$$

From (A. 10) and (A. 11) we have

$$
\begin{gathered}
Z_{p} \Pi_{Z_{f}}=\left(L_{31} L_{41}^{T}+L_{32} L_{42}^{T}\right)\left(L_{41} L_{41}^{T}+L_{42} L_{42}^{T}\right)^{-1} \\
\cdot\left(L_{41} Q_{1}^{T}+L_{42} Q_{2}^{T}\right)
\end{gathered}
$$

Therefore, from (A. 10) and (A.12), and using the notation (11), we obtain

$$
Z_{p}-Z_{p} \Pi_{Z_{f}}=\left[\begin{array}{ll}
P_{1} & P_{2}
\end{array}\right]\left[\begin{array}{c}
Q_{1}^{T} \\
Q_{2}^{T}
\end{array}\right]
$$

From (1), (A. 1), (A. 13) and

$$
\mathcal{Y}_{f}=L_{51} Q_{1}^{T}+\cdots+L_{55} Q_{5}^{T}
$$

in (10), we have

$$
\begin{gathered}
\Xi=\left(L_{51} P_{1}^{T}+L_{52} P_{2}^{T}\right)\left(P_{1} P_{1}^{T}+P_{2} P_{2}^{T}\right)^{-1} \\
\cdot\left(P_{1} Q_{1}^{T}+P_{2} Q_{2}^{T}\right)
\end{gathered}
$$

Hence, we conclude that

$$
\begin{aligned}
\Upsilon= & \Xi \Xi^{T} \\
= & \left(L_{51} P_{1}^{T}+L_{52} P_{2}^{T}\right)\left(P_{1} P_{1}^{T}+P_{2} P_{2}^{T}\right)^{-1} \\
& \quad \cdot\left(L_{51} P_{1}^{T}+L_{52} P_{2}^{T}\right)^{T} . \\
& \text { References }
\end{aligned}
$$

\section{References}

1) M. Verhaegen and P. Dewilde: Subspace model identification Part I: The output-error state space model identification class of algorithms, Int. J. Control 56-5, 1187/1210 (1992)

2) M. Verhaegen and P. Dewilde: Subspace model identification Part II: Analysis of the elementary output-error state-space model identification algorithm, Int. J. Control 56-5, 1211/1241 (1992)

3) M. Verhaegen: Subspace model identification Part 3: Analysis of the ordinary output-error state-space model identification algorithm, Int. J. Control 58-3, 555/586 (1993)

4) M. Verhaegen: Identification of the deterministic part of MIMO state space models given in innovations form from 
input-output data, Automatica 30-1, 61/74 (1994)

5) M. Jansson: Subspace identification and ARX modeling, in Proceedings of the 13th IFAC Symposium on System Identification, Rotterdam, The Netherlands, 1625/1630 (2003)

6) M. Verhaegen: Application of a Subspace Model Identification Technique to Identify LTI Systems Operating in Closed-loop, Automatica 29-4, 1027/1040 (1993)

7) T. Katayama, H. Kawauchi and G. Picci: Subspace identification of closed loop systems by the orthogonal decomposition method, Automatica 41-5, 863/873 (2005)

8) H. Oku and T. Fujii: Direct subspace model identification of LTI systems operating in closed-loop, in Proceedings of the 43rd IEEE CDC, Paradise Island, Bahamas, 2219/2224 (2004)

9) P. M. J. van den Hof and R. J. P. Schrama: An indirect method for transfer function estimation from closed loop data, Automatica 29-6, 1523/1527 (1993)

10) K. Peternell, W. Scherrer and M. Deistler: Statistical analysis of novel subspace identification methods, Signal Processing 52, 161/177 (1996)

11) L. Ljung: System Identification, 2nd Ed., Prentice Hall (1999)

12) P. Van Overschee and B. De Moor: Subspace Identification for Linear Systems, Kluwer Academic Publishers (1996)

13) B. Haverkamp and M. Verhaegen: SMI Toolbox Version 1.0., TUD/ET/SCE96.015 (1997)

14) M. Verhaegen and E. Deprettere: A fast, recursive MIMO state space model identification algorithm, inProceedings of the 30th IEEE CDC, Brighton, 1349/1354 (1991)

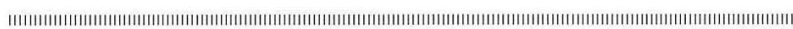

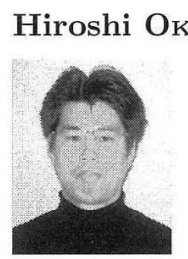

(Member)

He received the Ph.D. degree in Engineering from the University of Tokyo in 2000. In 1998-2000, he was a JSPS Research Fellow. In 2000-2002, He was a postdoctoral researcher in University of Twente. Currently, he works as Lecturer for Department of Engineering, Osaka Institute of Technology. He is a member of IEEE and ISCIE. He received Outstanding Paper Award and Sunahara Prize from ISCIE in 2005. His current research interests include system identification and change detection.

\section{Yasuko Ogura}

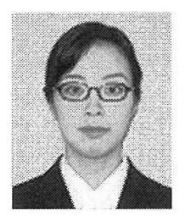

She received the MS degree in Engineering from Osaka Institute of Technology in 2006. Currently, she works for Mitsubishi Automotive Engineerig Co., Ltd. Her research interests include system identification.

\section{Takao FujII (Member)}

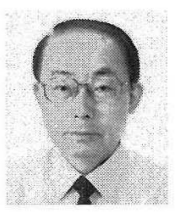

He recieved his M.S. degree and Dr. of Engineering from Osaka. University in 1969 and 1983, respectively. From 1990 to 1995, he was a Professor of Kyushu Institute of Technology. From 1995 to 1996, he was a Professor of Faculty of Engineering Science, Osaka University. Since 1997, he has been a Professor of Graduate School of Engineering Science, Osaka University. In 1987, he was awarded a prize of the paper on transaction of SICE, and in 1999, he was awrded a prize of the paper on transaction of ISCIE. His research interest are robust control, system theory and its applications. Dr. Eng. He is a member of ISCIE and IEEE.

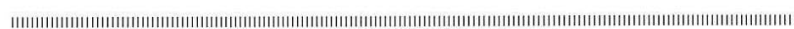

